\title{
分子印迹材料的先进制备技术与策略
}

\author{
王晓艳 ${ }^{1,2 *}$, 李金花 ${ }^{2}$, 陈令新 ${ }^{2,3^{*}}$ \\ 1. 滨州医学院药学院, 烟台 264003 ; \\ 2. 中国科学院烟台海岸带研究所, 中国科学院海岸带环境过程与生态修复重点实验室, 烟台 264003; \\ 3. 青岛海洋科学与技术试点国家实验室, 海洋生物学与生物技术功能实验室, 青岛 266237 \\ * 联系人, E-mail: wangxy@yic.ac.cn; 1xchen@yic.ac.cn
}

2018-09-21 收稿, 2018-12-17 修回, 2018-12-28 接受, 2019-02-26 网络版发表

国家自然科学基金(21804010,21876199，41776110)、山东省重点研发计划(GG201709290055)和山东省自然科学基金(ZR2014BL031)资助

摘要分子印迹聚合物(molecularly imprinted polymers, MIPs)是采用分子印迹技术(molecular imprinting technology，MIT)制备的，具有与模板分子在形状、大小及官能团方面完全匹配的特异识别位点的高分子聚合物， 能选择性识别和富集目标分析物(模板分子), 已广泛用于样品前处理、化学/生物传感等领域. 然而，在MIPs制备 和使用过程中，仍存在模板分子洗脱困难、有效识别位点少、结合容量低、传质速率慢、水相识别差等问题. 通 过借鉴融合其他领域的先进技术和策略, MIT发展迅速, 各种新型的印迹技术和策略不断涌现, 不仅有效解决了上 述问题，而且推动了新型MIPs的发展并拓展了其应用范围。本文以MIPs在样品前处理、传感和刺激响应中的应用 为导向，梳理了MIPs材料的先进制备技术(表面印迹和纳米印迹技术、可控/活性聚合技术、点击化学、固相合成 技术等)、策略(多模板、多功能单体、虚拟模板、片段印迹、嗍亲和印迹策略等)与刺激响应印迹(磁、温度、光 和 $\mathrm{pH}$ 响应等), 并对印迹技术和材料的发展进行了展望.

关键词分子印迹聚合物, 印迹技术, 印迹策略, 样品前处理, 传感, 刺激响应印迹

分子印迹技术(molecular imprinting technology, MIT) 是在模拟自然界中抗原-抗体间特异结合基础上 发展的，用于制备对模板分子具有特异性识别的聚合 物即分子印迹聚合物(molecularly imprinted polymers, MIPs)的技术. 1931年Polyakov和Khim ${ }^{[1]}$ 提出了 “具有 特殊吸附性能的硅胶粒子”，分子印迹开始萌芽. 1949 年, Dickey ${ }^{[2]}$ 首次提出“分子印迹”的概念. 然而, MIT在 Wulff等人 ${ }^{[3,4]}$ 成功制备了能够选择性识别糖类的共价 印迹聚合物后, 才有了跨越性的突破. 1993年Vlatakis等 人 $^{[5]}$ 在Nature 上发表了使用非共价法制备茶碱MIPs的 研究报道, MIT引起广泛关注和研究, 蓬勃发展为高分 子科学、材料学、生物和化学等交叉的新兴领域之一. 与天然的或其他方法制备出的识别材料相比, MIPs因
具有构效预定性、特异识别性和广泛实用性三大优点, 已被大量用于样品前处理、传感分析、模拟酶催化、 药物输送等领域 ${ }^{[6]}$.

目前用于MIPs制备的方法主要包括本体聚合、悬 浮聚合、乳液聚合、种子聚合、沉淀聚合等的自由基 聚合法, 以及溶胶-凝胶法. 然而, (单一)使用这些传统 方法制备的MIPs常具有诸多问题，如模板泄漏、吸附 容量低、形貌不规则、传质速率慢和亲水性差等，极 大地阻碍了MIPs的应用. 随着聚合物技术、纳米技术 和分析科学、环境科学、生命科学等学科的发展，通 过借鉴和融合其相关技术和策略，作为一门多学科交 叉技术的MIT也迅速发展. 各种巧妙的印迹技术(如表 面印迹、纳米印迹技术、可控/活性自由基聚合、中空

引用格式: 王晓艳, 李金花, 陈令新. 分子印迹材料的先进制备技术与策略. 科学通报, 2019, 64: 1352-1367 Wang X Y, Li J H, Chen L X. Advanced preparation technologies and strategies for molecularly imprinted materials (in Chinese). Chin Sci Bull, 2019, 64 1352-1367, doi: 10.1360/N972018-00964 
聚合物合成、点击化学环加成反应及固相合成技 术)、特殊的印迹策略(如多模板、多功能单体、虚拟 模板、片段印迹、嗍亲和印迹策略)和刺激响应印迹 (如磁、温度、光和 $\mathrm{pH}$ 响应) 不断涌现 ${ }^{[7]}$. 在传统印迹方 法基础上, 借用和组合这些新颖的印迹技术/策略, 大大 加速了MIT的发展、有效改善了MIPs的使用效率和拓 展了其应用范围. 根据样品前处理、传感(图1)和刺激 响应应用的不同，本文综述了MIPs材料的先进制备技 术与策略，探讨了如何选择合适的印迹技术/策略来解 决关键问题、获得MIPs的优异性能等，并对印迹技术 和材料的发展进行了展望.

\section{1 面向样品前处理应用的印迹技术和策略}

MIPs在样品前处理中的应用，主要是作为选择性 的吸附剂与各种前处理技术联用, 如固相萃取 (solidphase extraction, SPE)、分散固相萃取(dispersive SPE, DSPE)、磁固相萃取(magnetic SPE, MSPE)、固相微萃
取和搅拌棒吸附萃取等. 为提高萃取效率, 对吸附剂材 料的要求是吸附量大、选择性高、均匀性好、亲水性 好等，这就需要在传统制备方法的基础上引人新的印 迹技术与策略. 主要包括表面印迹、纳米印迹技术及 可控活性自由基聚合、点击化学、中空多孔聚合物制 备、固相合成等技术，以及多模板、多功能单体、虚 拟模板/片段印迹、磁材料印迹和嗍亲和印迹等策略 (图2)，表面印迹和纳米印迹技术通常与其他印迹技术/ 策略结合使用.

\section{1 面向样品前处理应用的印迹技术}

传统的自由基聚合由于链增长速率不易控制，导 致制备的印迹聚合物往往粒径尺度分布比较大，而且 形貌不规则 ${ }^{[8]}$. 可控/活性自由基聚合(controlled/living free radical polymerization, CLRP)技术具有无终止、 无转移、引发速率远大于链增长速率等优点, 可以使 体系中的自由基浓度控制得很低而且抑制自由基双基

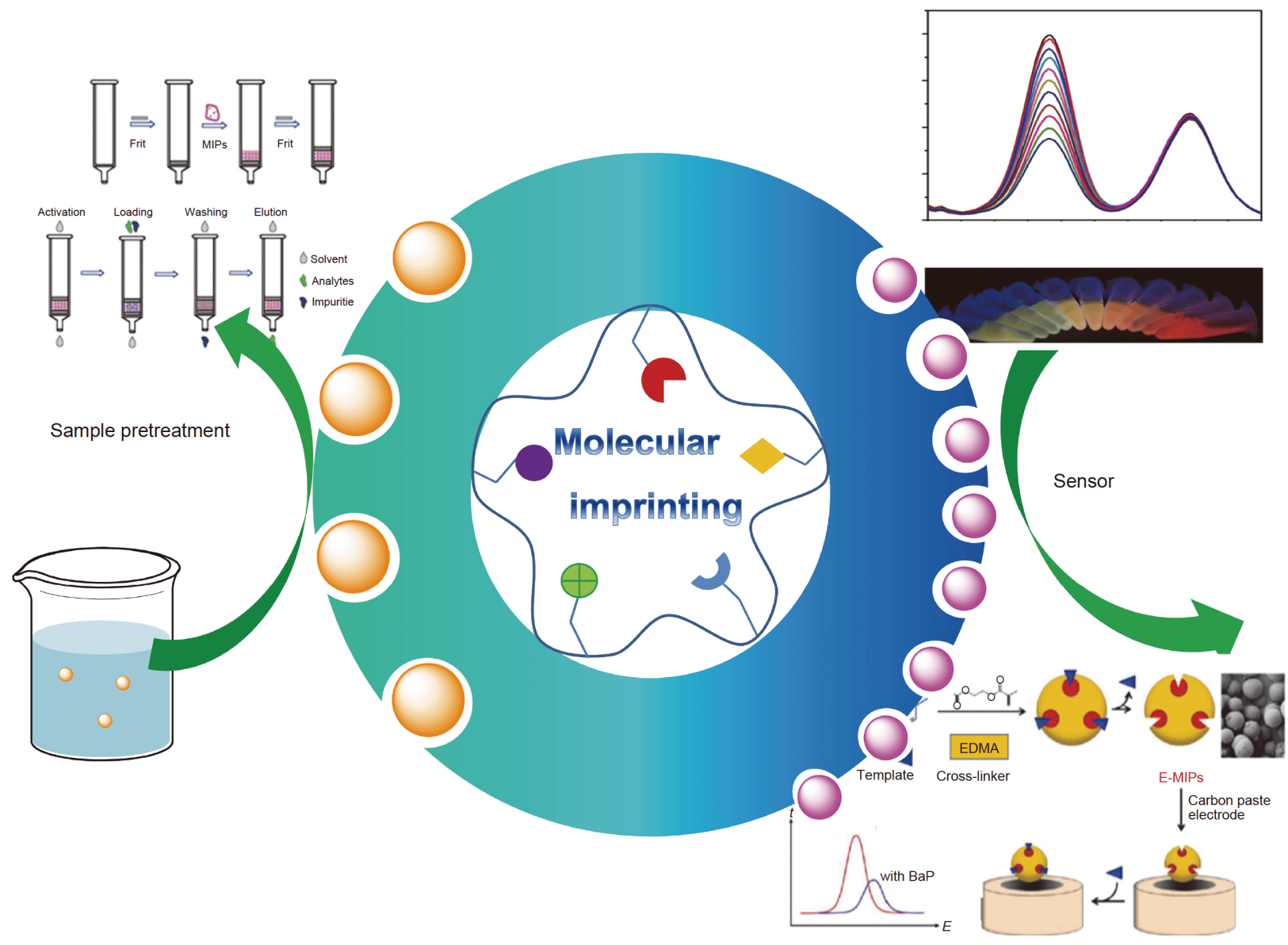

图 1 (网络版彩色)MIPs在样品前处理和传感中的应用

Figure 1 (Color online) Application of MIPs in sample pretreatment and sensors 


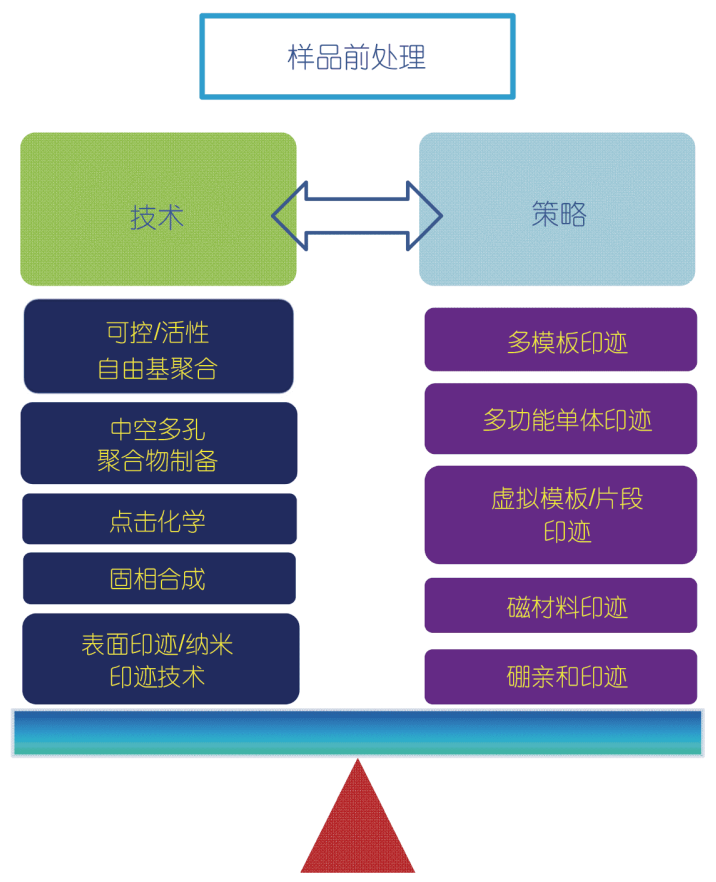

图 2 (网络版彩色)面向样品前处理应用的印迹技术和策略 Figure 2 (Color online) Imprinting technology and strategy for sample pretreatment application

终止, 进而控制聚合物的分子量和分子量分布, 是改善 聚合物粒度分布和形貌规则的重要手段 ${ }^{[9]}$. 其中以原子 转移自由基聚合(atom transfer radical polymerization, ATRP)和可逆加成-断裂链转移(reversible addition-fragmentation chain transfer, RAFT)聚合最为常用. Beyazit 等人 ${ }^{[10]}$ 对CLRP在MIPs制备中的应用, 进行了综述. 对 于ATRP法，可用于在不同载体上固定引发剂，用最简 便的印迹聚合物合成条件合成MIPs. 然而，最大的局 限性是在合成MIPs时，功能单体的选择非常有限：典 型的酸性功能单体如甲基丙烯酸与ATRP不相容, 碱性 的功能单体如甲基丙烯酰胺和乙烯基吡啶很难获得高 的单体转换率 ${ }^{[10,11]}$. RAFT能与大多数的功能单体兼容, 是目前应用最广泛的CLRP技术. 此外, 由于RAFT的功 能基团能接枝到聚合物上，通过RAFT能制备具有亲水 性和窄分散性的印迹聚合物 ${ }^{[12 ~ 14]}$.

点击化学(click chemistry)由Kolb等人 ${ }^{[15]}$ 在2001年 率先使用，是另一种控制聚合物大分子结构的制备技 术, 因制备条件温和、快速和高效而受到广泛关注. 然 而, 直到2010年, 点击化学才应用到MIPs的制备中, 主 要通过铜催化叠氮化物-炔烃环加成反应，在聚合物表 面引人功能链, 对材料进行改性和增加新的功能 ${ }^{[16]}$. Bonomi等人 ${ }^{[17]}$ 在纯水中利用点击化学制备了印迹聚合
物，该印迹聚合物具有很好的生物相容性. Awino等 人 $^{[18]}$ 利用点击化学, 通过铜催化叠氮化物-炔烃环加成 反应, 以4-乙烯基苯硼酸为功能单体, 制备了亲水性的 印迹胶束(图3). 该胶束能精确定位结合基团, 甚至能区 分糖类物质的细微结构变化.

中空多孔聚合物制备技术(hollow porous polymer synthesis technology)的优势是能提高印迹材料的结合 容量和加快目标分子传质速率. 中空多孔印迹材料的 制备一般以 $\mathrm{SiO}_{2}$, 介孔 $\mathrm{SiO}_{2}$, 聚苯乙烯微球等为支撑 体 $^{[19 \sim 26]}$, 在其表面制备印迹层, 通过氢氟酸(HF)或四氢 呋喃(THF)将内核 $\mathrm{SiO}_{2}$ 或聚苯乙烯微球溶掉，以制得中 空多孔的印迹材料. Ostovan等人 ${ }^{[20]}$ 以介孔 $\mathrm{SiO}_{2}$ 为支撑 材料, 通过表面印迹技术制备了格列本艮印迹层, 然后 用HF将支撑体溶掉，获得了中空多孔的印迹壳层材料 (图4). 该材料相比于相应的核-壳印迹材料(传质速率 $>60 \mathrm{~min}$, 吸附容量 $\left.29.8 \mathrm{mg} \mathrm{g}^{-1}\right)$, 具有更快的传质速率 (<30 min)和更高的吸附容量 $\left(50.0 \mathrm{mg} \mathrm{g}^{-1}\right)$. 这主要归功 于中空多孔的印迹材料其分子识别位点仅分布于壳层 材料上, 便于模板分子的进出, 在提高传质速率的同时 也增大了单位质量MIPs的结合容量.

固相合成(solid-phase synthesis)是一种新型MIPs制 备技术，它是将模板分子接枝在固体载体基质表面以 制备固定模板，然后将固定模板放置在含有单体、交 联剂和引发剂的反应器中进行聚合, 最后在固定模板 表面上制备印迹聚合物. 该法的优点是所有的识别位 点位于表面且具有相同的取向，这样可大大提高识别 位点的均匀性，有利于模板分子的结合并增强材料的 吸附性能 ${ }^{[27]}$. 固相载体的选择一般是玻璃珠 ${ }^{[28 ~ 31]}$, 也 可以是石英芯片 ${ }^{[32]}$. 然而, 由于商业化的玻璃珠尺寸较 大，一般在70 100 $\mu \mathrm{m}$, 相对较大的尺寸导致较低的比 表面积, 使得固定在其表面的模板分子数量减少, MIPs 产率很低(每克玻璃珠制备MIPs 为 $0.15 \sim 0.3 \mathrm{mg}$ ). 基于 此, Ashley等人 ${ }^{[33]}$ 提出一种新的分散固相印迹方法, 以 磁性 $\mathrm{Fe}_{3} \mathrm{O}_{4}$ 纳米粒子为固相载体, 由于纳米粒子具有较 高的比表面积，所以制备的MIPs的产率大大提高，是 传统用玻璃珠制备的83 167倍. 固相合成技术的高产 率及可扩展性高、重现性好、可控性好、易于自动化 等优点, 有望推动MIPs的规模化生产与应用 ${ }^{[34]}$.

\section{2 面向样品前处理应用的印迹策略}

样品前处理技术在消除基质干扰、萃取富集痕量 目标物方面发挥了重要作用. 然而, 样品前处理操作仍 

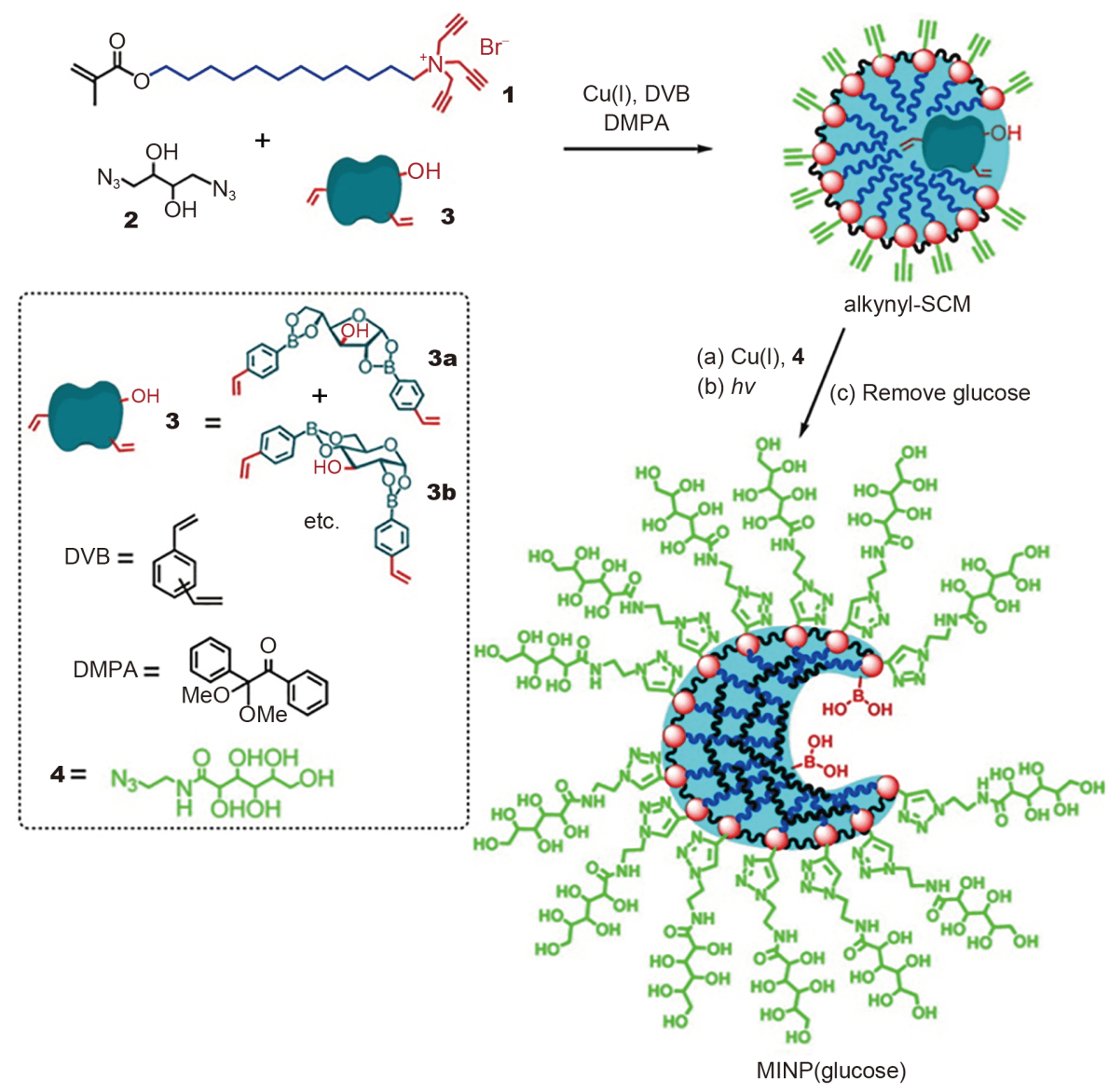

图 3 (网络版彩色) 硼酸功能化的分子印迹纳米粒子 (MINP) ${ }^{[18]}$

Figure 3 (Color online) Preparation of boronic acid-functionalized MINP ${ }^{[18]}$
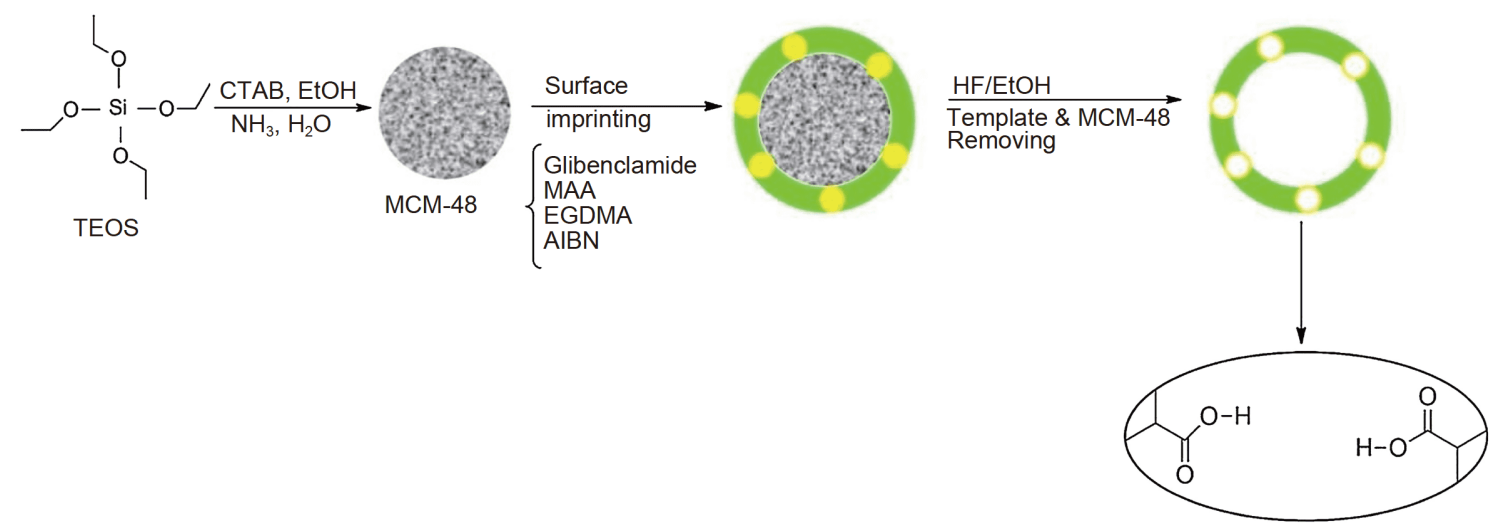

图 4 (网络版彩色)中空多孔分子印迹聚合物制备过程 ${ }^{[20]}$

Figure 4 (Color online) Preparation of hollow porous MIPs ${ }^{[20]}$

存在步骤烦琐、试剂消耗多、耗时费力的问题. 因此, 目前迫切需要发展具有高选择性、操作简单、省时省 力的样品前处理技术，同时对作为吸附剂材料的MIPs 提出更高的要求. 多模板、多功能单体、虚拟模板、
片段印迹及磁材料印迹、嗍亲和印迹等先进策略应运 而生.

为了能够选择性同时识别多种目标物，提高MIPs 的利用率和节省前处理时间, 发展了多模板印迹(multi- 
template imprinting)策略, 即以两个或多个目标物为模 板制备MIPs材料, 在一个印迹聚合物材料中含有多个/ 种模板分子的识别位点, 能够实现多种物质的同时富 集、分离, 适于多残留、高通量分析. 例如, 本课题 组 ${ }^{[35]}$ 采用多模板印迹策略以 16 种多环芳烃 $(\mathrm{PAHs})$ 为模 板分子，结合表面印迹技术结合溶胶-凝胶聚合制备了 多模板MIPs(mt-MIPs)材料. 该材料对 16种PAHs表现出 优异的吸附容量和显著的结合选择性, 将其作为SPE吸 附剂并结合GC-MS实现了对海水中16种PAHs的同时 高效地识别、萃取、检测和去除. 本课题组 ${ }^{[36]}$ 还以6种 酚类化合物的混合物作为模板，一步沉淀聚合制备了 mt-MIPs用作SPE填料，结合毛细管电泳，同时、高选 择、快速地萃取富集和测定了工业废水、河水等水样 中的6种酚类化合物. 近来, 本课题组 ${ }^{[37]}$ 又以 3 种维生素 $\mathrm{B}$ 为模板分子, 以葡萄糖为碳源制备碳球，使用壳聚糖 兼做功能单体与交联剂，在乙酸的水溶液中室温制得 mt-MIPs, 将其用于DSPE吸附剂, 结合HPLC-UV, 实现 了对橘汁中 3 种维生素B的同时、快速地识别、萃取和 检测. 同时, 也应该指出, mt-MIPs的选择性(对某一模 板分子来说)相较于单一模板MIPs降低, 这很可能是由 于每种模板的结合位点数目被稀释及多种模板增加的 再混合效应导致的. 事实上, mt-MIPs的印迹效应是多 种模板分子的印迹性能的平衡、妥协的综合结果.

在印迹过程中使用多功能单体(multi-functional monomer imprinting)对于提高印迹材料对模板分子的 选择性是不错的选择, 即通过使用多种功能单体与模 板分子不同识别位点作用, 发挥多功能单体协同作用, 增加识别位点数量, 从而提高MIPs的识别能力和选择 性. 例如, $\mathrm{Xu}$ 等人 ${ }^{[38]}$ 采用多功能单体印迹策略, 以牛血 清和多巴胺为双功能单体, 制备了四环素MIPs, 该材料 对四环素有较高的选择性, 能直接从牛奶样品中选择 性地分离四环素. Liao等人 ${ }^{[39]}$ 以甲基丙烯酸(MAA)和 丙烯酰胺(AA)为双功能单体，制备了奥硝唑印迹聚合 物，由于酸性MAA的差基易与硝基咪唑环上氮形成氢 键，碱性AA的酰氨基易与硝基咪唑上羟基等形成氢键, 功能单体优势互补, 提高了聚合物对模板分子的吸附 性. 制备的双功能单体MIPs相较单一功能单体MIPs在 吸附容量、选择性等方面有明显优势. 的确, 使用多功 能单体是提高选择性的好途径，也是印迹各种分析物 尤其是生物大分子的有效方式. 然而, 如何合适地选择 及合理地组合现有的多种功能单体，如何巧妙设计合 成新功能单体, 并进一步有效利用它们的协同效应, 都
极具挑战.

虚拟模板印迹(dummy imprinting)是使用在结构、 形状和大小上与目标分子相似的分子作为模板进行印 迹; 片段印迹(segment/fragment imprinting)是使用目标 分子中含有特定官能团的一部分(片段结构)作为虚拟 模板进行印迹，通过对片段的识别达到对整个分子的 识别. 若根据一类目标物质的共有结构, 篮选出理想的 模板分子用于制备虚拟/片段MIPs，即可实现对一类具 有共同结构的物质的选择性富集和同时分离测 定 $^{[40-42]}$. 这两种策略除了可以有效避免模板泄漏造成 的污染及其对检测结果的干扰从而解决模板泄漏的问 题 ${ }^{[40,43 ~ 49]}$ ，尤其适用于目标分子造价昂贵、易燃易 爆、易降解、溶解度过低等不适合作为模板分子的情 况 $^{[50 \sim 53]}$. 如Özer等人 ${ }^{[41]}$ 以邻苯二甲酸二乙酯为虚拟模 板，制备了虚拟印迹聚合物(DMIPs)，结合GC-MS，实 现了水样中 6 种邻苯二甲酸酯的同时检测. $\mathrm{Xu}$ 等人 ${ }^{[52]}$ 以 三硝基苯酚(TNP)为虚拟模板，采用溶胶-凝胶聚合制 备了DMIPs, 实现了对土壤样品中三硝基甲苯(TNT)炸 药的高灵敏检测. Dan和Wang ${ }^{[53]}$ 使用双片段印迹策略 制备了MIPs检测软骨藻酸(DA), 使用DA的结构类似部 分戊烷-1,3,5-三羧基酸和脯氨酸作为虚拟模板替代毒 性大且价格昂贵的DA, 制备了双片段印迹MIPs, 对DA 具有高选择性. 需要指出的是, 尽管虚拟模板印迹和片 段印迹有许多优势，但是合适的虚拟模板和片段模板 的选择仍然是一个难点.

磁材料印迹(magnetic material imprinting, 同下文的 磁刺激响应印迹)策略是在印迹过程中引人磁性材料, 即将MIPs修饰在磁性材料(如 $\mathrm{Fe}_{3} \mathrm{O}_{4}$ 磁性微球)表面形成 分子印迹磁性材料(MMIPs). 这种材料在使用时通过磁 性分离就可将吸附了目标化合物的微球和样品基质快 速分离, 使整个样品前处理过程变得简单易行, 同时也 避免了残留吸附剂造成的二次污染问题 ${ }^{[7]}$. 最近, 欧阳 罡峰课题组 ${ }^{[54]}$ 详细总结了各种MMIPs的制备及其在 MSPE等样品前处理方面的应用, Ansari ${ }^{[55]}$ 也对MMIPs 的合成及用于食品和环境分析中的MSPE和分离进行 了综述. 简单、快速、低成本且环境友好的MIPs磁萃 取方法在复杂基质痕量分析中应用潜力巨大.

嗍亲和印迹(boronate affinity imprinting)是在印迹 过程中, 使用含有嗍酸配基的功能单体, 利用嗍酸配基 与目标分子中的顺式二差基基团间进行的可逆结合, 在 $\mathrm{pH} \geqslant 8.5$ 时, 嗍酸配基中的嗍原子与氢氧根结合, 生 成嗍酸负离子, 能与顺式二差基基团发生酯化反应, 得 
到五元或六元环状复合物. 在酸性条件下该环状酯解 离，释放出含顺式二羟基的分子; 利用这一策略，可以 对含有顺式二羟基基团的小分子、单糖、多糖及糖蛋 白进行识别. 刘震课题组 ${ }^{[56 r 60]}$ 基于硼亲和作用制备了 系列印迹材料，用于糖基化和磷酸化等翻译后修饰蛋 白质样品的富集等. 为了解决蛋白质印迹的困难, 该课 题组 ${ }^{[60]}$ 提出光刻硼亲和分子印迹法, 以4-乙烯基苯硓 酸为功能单体, 在弱碱性条件下, 模板蛋白与功能单体 通过嗍亲和作用形成复合物，与交联剂在光引发剂作 用下进行聚合, 酸性条件将模板洗脱, 得到MIPs, 由于 光聚合的时间较短，有效避免了因蛋白失活而造成印 迹失败. 该法具有很好的普适性，更换模板糖蛋白时， 只需微调功能单体、交联剂和致孔剂的比例及控制反 应的时间. 该印迹材料具有专一识别性和高亲和性, 通 过与酶联免疫分析联用，成功用于人血清中甲胎蛋白 的检测. 然而这种方法仅适合光能够照射到的表面，因 此制备的印迹层很薄. 在此基础上, 该课题组 ${ }^{[61 ~ 63]}$ 进一 步发展出硼亲和可控定向表面印迹策略. 如图5所示, 首先将模板分子固定到硼酸功能化的基质上，然后选 用生物相容性良好的功能单体在基质表面聚合，制备 出厚度合适的印迹层以及和模板分子空间匹配的印迹 空腔 ${ }^{[64]}$. 该方法的最大优势在于印迹过程可控: 根据模 板分子尺寸的大小，通过调节印迹时间来精确控制调 整印迹层厚度, 制备不同尺寸模板的分子印迹层. 该方 法不仅简化了印迹步骤，而且适用范围广，能够便捷、 高效地制备出糖蛋白、聚糖和单糖的MIPs. 同时, 为了 获得更好的实际应用，该课题组 ${ }^{[65]}$ 将硼亲和可控定向
表面印迹法与96孔板联合使用，将制备的MIPs取代吸 附在 96 孔板上的抗体, 进行了酶联免疫夹心实验, 实现 了人血清中甲胎蛋白的检测，该测试结果与放射免疫 分析法测得的结果一致.

此外, 刘震课题组 ${ }^{[6,58,66]}$ 还提出了双模板对接定向 印迹法. 如图6所示，首先以溴化十六烷基三甲基铵 $(\mathrm{CTAB})$ 为模板, 制备介孔 $\mathrm{SiO}_{2}$, 因带正电荷, 能与带负 电的印迹模板通过静电作用形成双模板复合物，然后 在功能单体 $\gamma$-氨丙基三乙氧基硅烷(APTES)、含有硓 酸配基的硅烷化试剂及交联剂四乙氧基硅烷(TEOS)作 用下聚合, 洗脱模板, 得到MIPs ${ }^{[66]}$. 该策略的核心是: 模 板-模板对接定向印迹. 印迹方法简单且印迹效率高, 印 迹材料主要用于腺苷酸、磷酸肽等的富集.

\section{2 面向传感应用的印迹技术和策略}

MIPs在传感中的应用主要是作为传感器的识别单 元, 特异性的结合目标分子后输出检测信号, 也就是说 MIPs在分子印迹传感器中兼具识别与转导功能. 根据 转导机理不同，输出的检测信号主要分为电化学、光 学、压电3种类型；其中，分子印迹电化学、苂光和表 面增强拉曼散射传感器是研究热点. 在传感应用时, 通 常要考虑传感器的响应时间、线性范围、灵敏度、选 择性及重现性等参数. 为此，在传统制备方法基础上, 有必要引人先进的印迹技术/策略来构建新型的印迹传 感器. 纳米印迹(nanoimprinting)技术、表面印迹(surface imprinting)技术和复合材料印迹(composite material imprinting)策略成为首选.

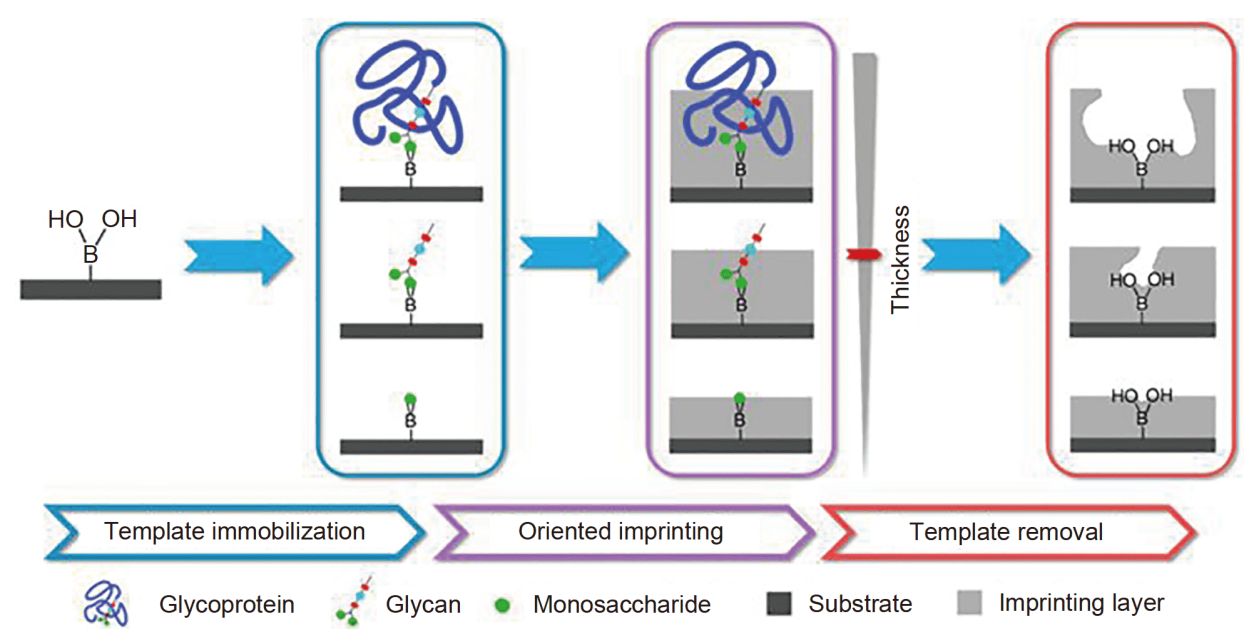

图 5 (网络版彩色)硼亲和可控定向表面印迹法 ${ }^{[64]}$

Figure 5 (Color online) Schematic of boronate affinity controllable-oriented surface imprinting ${ }^{[64]}$ 


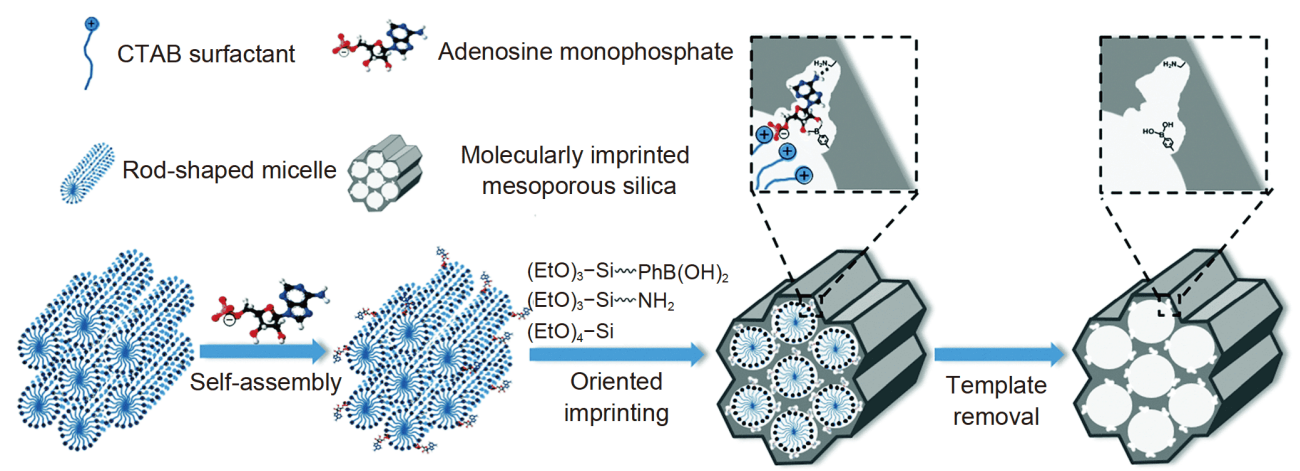

图 6 (网络版彩色)双模板对接定向印迹法 ${ }^{[66]}$

Figure 6 (Color online) Schematic of the dual-template docking oriented molecular imprinting ${ }^{[66]}$

纳米印迹(nanoimprinting)技术是将纳米材料的制 备技术引人到印迹材料的制备过程中，或是直接使用 纳米材料参与印迹. 由于纳米尺度材料能够极大提高 材料的比表面积, 采用纳米印迹技术, 将会大大提高基 于分子印迹的传感器的检测灵敏度、获得低的检测限 和宽的线性范围. 对于分子印迹电化学传感器, 主要是 用纳米材料修饰电极，然后在电极表面制备分子印迹

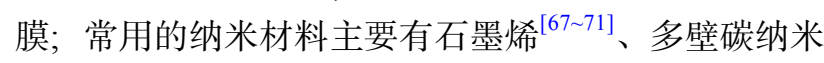
管 ${ }^{[72 \sim 74]}$ 、量子点 ${ }^{[75 \sim 77]}$ 和金纳米粒子 ${ }^{[78 \sim 81]}$ ，这些纳米材 料具有很高的比表面积、可调的表面性质及优异的导 电性能, 极大地提高了传感器的灵敏度.

近年来, 分子印迹荧光传感器发展迅速, 兼具MIPs 的高选择性和苂光检测的高灵敏度，本文将着重介绍 分子印迹苂光传感器的构建方法和策略. 分子印迹苂 光传感器除了将纳米材料, 如量子点 ${ }^{[22,83]}$ 、碳点 ${ }^{[84,85]}$ 、 金属纳米簇 ${ }^{[86]}$ 、上转换纳米粒子 ${ }^{[87,88]}$ 引人印迹过程外, 在印迹的过程中还要使用纳米材料的制备技术，制备 出纳米球、纳米膜、核-壳纳米球等印迹材料, 解决传 统方法制备的尺寸大、识别位点深埋于印迹材料内部 导致的传质速率慢进而影响灵敏度的问题 ${ }^{[89]}$. 基于纳 米球的核-壳型MIPs是纳米印迹技术和表面印迹(surface imprinting)技术相结合的产物,一般选用纳米粒子, 如 $\mathrm{SiO}_{2}{ }^{[90]}, \mathrm{Fe}_{3} \mathrm{O}_{4}{ }^{[91]}$ 和量子点 ${ }^{[65,92,93]}$ 等作为核支撑材料, 在其表面进行印迹，制备的印迹层在几个至十几个纳 米厚度范围内, 能将模板分子完全去除, 由于识别位点 位于表面, 方便目标分子的结合, 具有较快的传质速率, 特别适用于传感分析.

对于分子印迹荧光传感器，最常见的是单一苂光 信号的猝灭型. 但是, 单一信号尤其是猝灭型苂光传感 器，苂光信号的变化容易受检测物浓度、苂光材料自
身浓度、外部环境及检测设备条件变化等多种因素的 影响, 导致测量值与真实值之间存在一定的误差. 近年 来, 比率苂光法发展迅速, 比率苂光是在单一波长激发 下，产生两个发射峰，加人目标物后，两处发射峰的苂 光强度比值会发生变化, 建立苂光强度比值与目标物 的线性关系, 以此测定目标物. 这种内在的自我校正可 降低甚至消除非目标因素的影响, 从而得到更为准确 的结果 ${ }^{[94]}$. 将比率苂光法引人到分子印迹传感器的制 备过程中, 制备分子印迹比率苂光传感器, 能增强检测 的灵敏度, 并可以对目标物进行可视化检测. 印迹比率 苂光传感器的构建需要运用复合材料印迹(composite material imprinting)策略, 如何保证各种纳米材料在印 迹过程中不影响各自的功能, 是制备此类传感器的一 大挑战.

基于荧光共振能量转移(FRET)机理的分子印迹比 率苂光传感器, 在制备过程中, 控制供体和受体距离至 关重要. 因此, 印迹层厚度的控制尤为重要(控制在 $10 \mathrm{~nm}$ 内)，所以纳米印迹技术是必需的; 同时，采用表 面印迹技术制备核-壳结构的印迹材料也是理想的选 择. 本课题组 ${ }^{[95]}$ 基于FRET原理，以苂光染料NBD为供 体苂光团, 藻蓝蛋白为受体苂光团, 以纳米 $\mathrm{SiO}_{2}$ 为核支 撑体，通过表面印迹技术制备了几个纳米厚度的印迹 层(图7), 当藻蓝蛋白与识别位点结合时, 供体NBD的 苂光发生猝灭, 而受体藻蓝蛋白的菼光得到增强, 通过 比率苂光值变化来定量检测藻蓝蛋白.

参比苂光比率型分子印迹苂光传感器, 是使用较 多的分子印迹比率苂光传感器，其印迹材料中含有两 种不同发射峰的苂光材料，其中一个发射峰的强度与 目标物有关, 而另一发射峰的强度保持不变作为参比, 这种构建策略与具有内标的分析方法相似. 在印迹过 

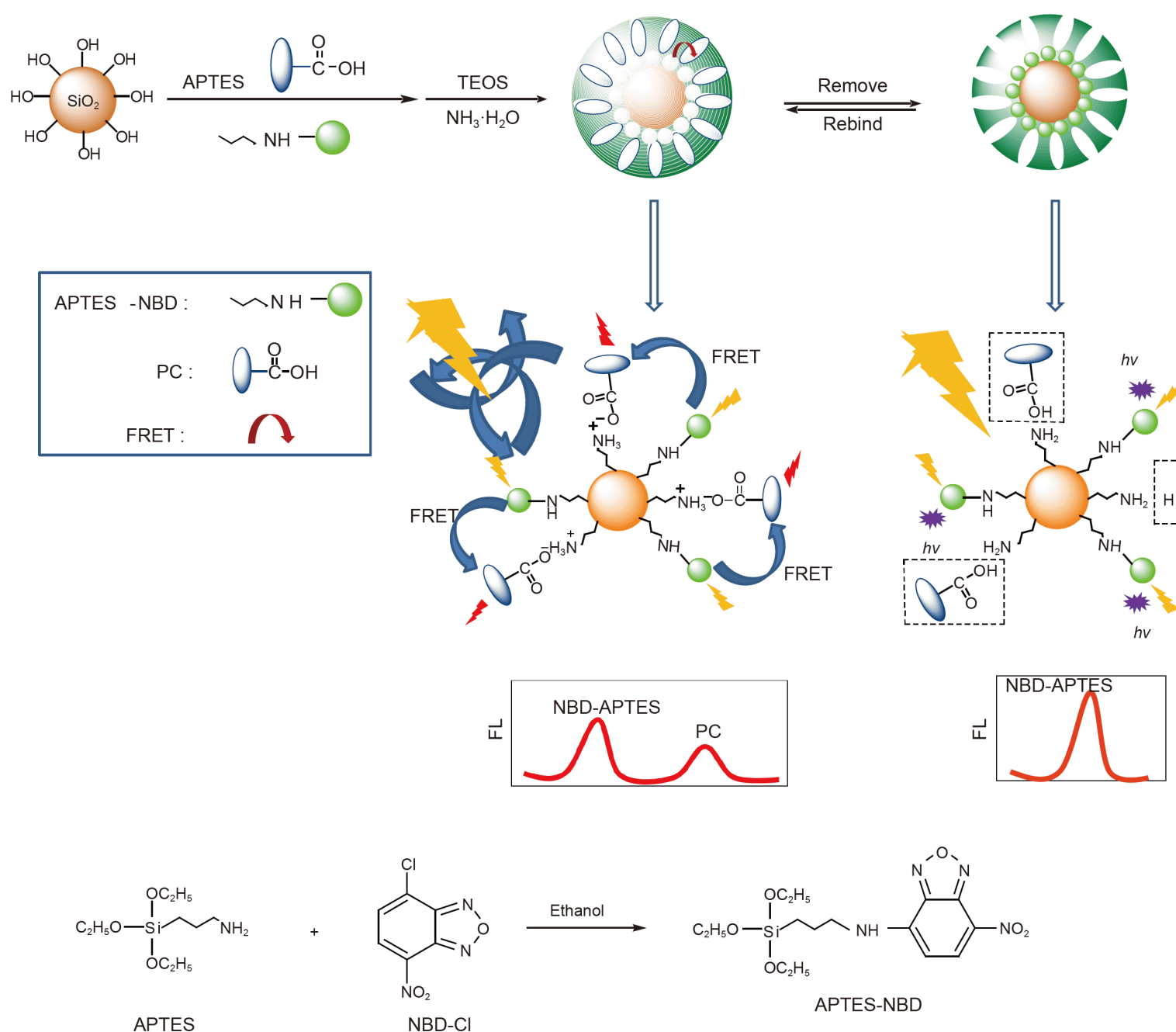

图 7 (网络版彩色)核-壳结构分子印迹比率苂光传感器的构建及可能的检测机理 ${ }^{[95]}$

Figure 7 (Color online) Schematic illustration for the preparation process and possible detection principle of core-shell structured molecular imprinting ratiometric fluorescent sensor ${ }^{[95]}$

程中, 如何保证参比苂光强度保持不变, 是必须考虑的 问题. 一般情况下, 采用包埋法将作为参比的苂光材料 (量子点、碳点、苂光染料等)与目标分子隔绝, 但又不 能影响其苂光发射. 例如, $\mathrm{SiO}_{2}$ 用作包埋材料是不错的 选择, 主要是因为它透光性强并具有一定的惰性, 能够 阻止内部包裹的苂光材料不与外部的目标物接触且能 够发射出苂光. 包覆苂光材料的 $\mathrm{SiO}_{2}$ 再作为核支撑材 料, 采用表面印迹技术, 在印迹过程中加人另一种苂光 材料(量子点、金纳米簇、碳点、苂光染料等)参与印 迹, 制备参比菼光比率MIPs传感器 ${ }^{[96-99]}$. 本课题组 ${ }^{[99]}$ 以 包覆红色量子点的 $\mathrm{SiO}_{2}$ 为核支撑材料, 红色量子点为 参比信号，绿色苂光染料NBD为识别信号, APTES为 功能单体，2,4-二氯苯氧乙酸(2,4-D)为模板分子, 通过
溶胶-凝胶法制备了印迹层(图8)，随着2,4-D浓度的增 大, 比率菼光强度也随之变化, 苂光颜色也由橙红色变 为绿色, 实现了 $2,4-\mathrm{D}$ 的可视化检测. 在印迹过程中也可 加人 CTAB，制备具有大孔隙体积的介孔材料，高度贯 通的纳米孔结构有助于提高目标分子的传质速率，从 而提高检测灵敏度 ${ }^{[100,101]}$. 此外, 使用 $\mathrm{SiO}_{2}$ 包埋苂光的 材料也可不参与印迹过程, 直接作为掺杂的参比, 在检 测过程中使用, 避免烦琐的优化印迹材料制备过程 ${ }^{102]}$.

目前, 表面增强拉曼散射(surface enhanced Raman scattering, SERS)类型的分子印迹传感器引起关注, MIT与SERS联用制备的印迹材料兼有特异识别性和高 灵敏度的优点. 刘震课题组 ${ }^{[103 ~ 106]}$ 将嗍亲和可控定向表 面印迹技术与金/银纳米粒子和等离激元材料等各种材 


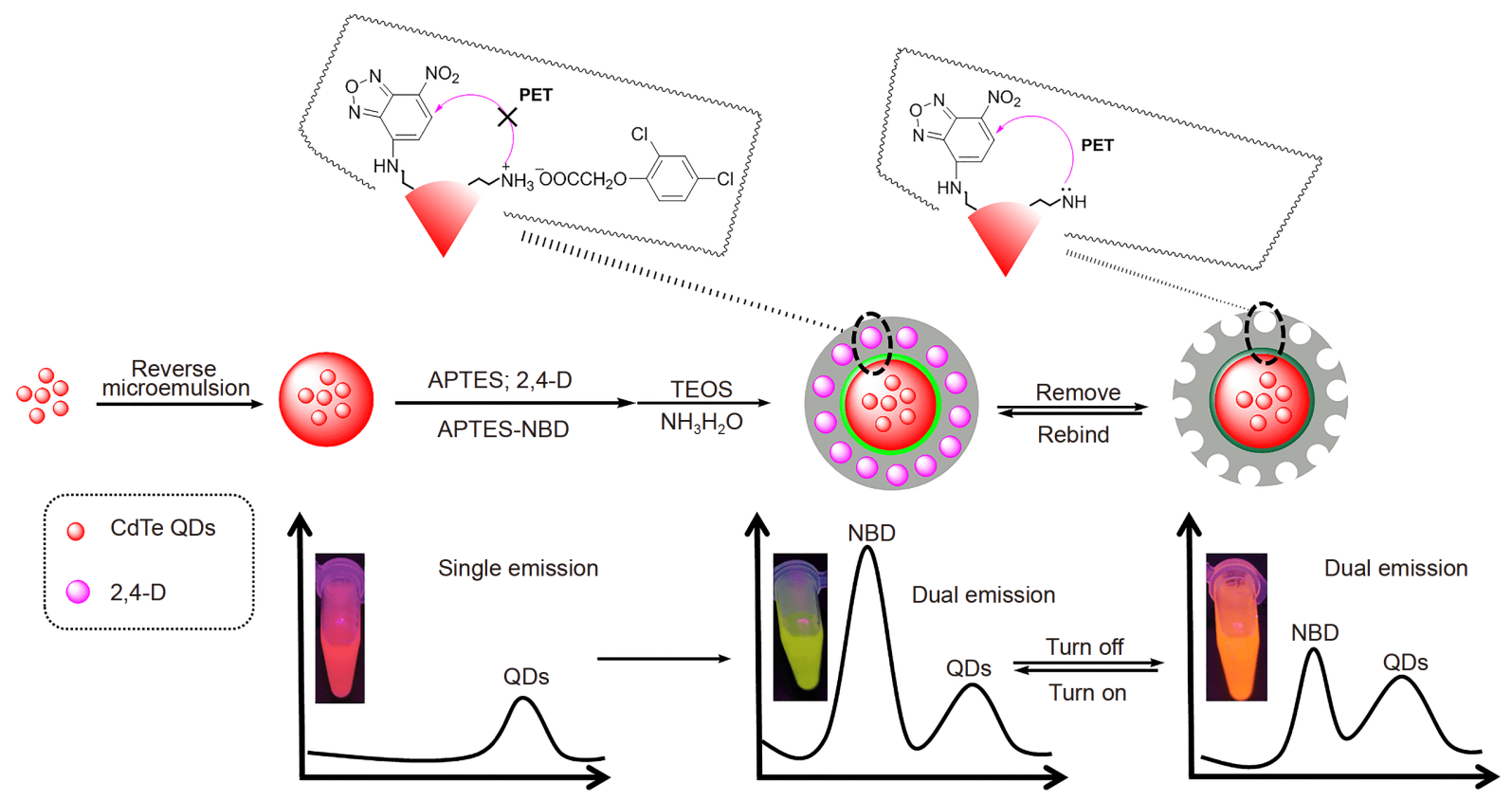

图 8 (网络版彩色)2,4-D比率印迹纳米球的制备过程和可能的检测原理 ${ }^{[99]}$

Figure 8 (Color online) Schematic illustration for the preparation process and possible detection principle of 2,4-D ratiometric imprinting nanoparticles ${ }^{[99]}$

料相结合, 制备了具有仿生功能的识别材料, 在疾病诊 断、癌细胞靶向识别和单细胞分析等领域已经获得重 要应用. 该课题组 ${ }^{[103 ~ 106]}$ 发展了表面等离激元免疫夹心 法(PISA), 采用活体微萃取和表面等离激元光学检测用 于检测单个活细胞和活体动物中的低拷贝数蛋白. 如 图9所示，在金基微萃取探针的表面修饰单克隆抗体或 MIPs, 然后在显微操作平台的辅助下插人到单细胞中. 在很短的时间内，目标蛋白质被专一高效地萃取到探 针表面. 微萃取探针拔出并清洗后, 将能识别目标蛋白 质的抗体或嗍亲和配基的银基纳米拉曼探针修饰到微 萃取探针上, 制备萃取探针-目标蛋白质-拉曼标签三明 治型复合物结构. 当用激光束照射该三明治型复合物
表面时, 产生表面等离激元光学效应, 银基拉曼标签输 出SERS信号，金基微萃取探针发生表面等离子波会进 一步增强银基拉曼标签的SERS信号，从而大大提高检 测灵敏度，检测限可达单分子水平. 该单细胞分析技术 在癌症诊断、癌症预后和细胞质量控制等领域的应用 前景广阔 ${ }^{[105]}$.

\section{3 面向刺激响应应用的印迹技术和策略}

刺激响应聚合物(SRPs)又称环境敏感型聚合物, 是 在特定条件下通过自身物理或化学性质的改变而对外 界刺激(磁、热、光、电、力; $\mathrm{pH}$ 、离子强度、各种化 学物质和生物物质等)做出敏锐的应答. 将刺激响应聚

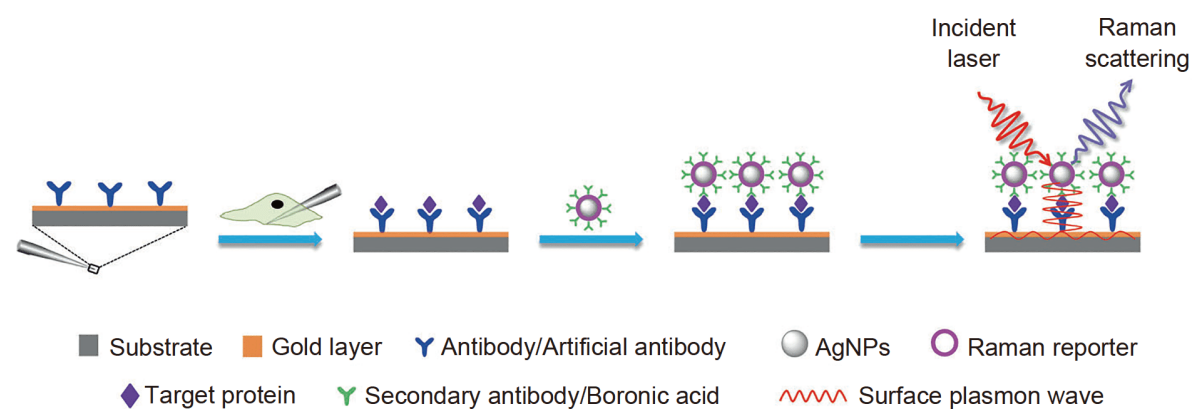

图 9 (网络版彩色)表面等离激元免疫夹心法的单细胞分析原理示意图 ${ }^{[105]}$

Figure 9 (Color online) Overview of probing protein expression in a single cell by plasmonic immunosandwich assay ${ }^{[105]}$ 
合物制备技术与MIT相结合制备的刺激响应MIPs(stimuli-responsive MIPs, SR-MIPs), 不仅具有SRPs响应外 界刺激的能力, 而且具有MIPs的分子识别能力, 更重要 的是这种高选择性识别能力受外界刺激的调控. 也就 是说，面向刺激响应应用的印迹技术和策略即刺激响 应印迹(stimuli-responsive imprinting), 是通过制备SRMIPs, 用于对目标分子的特异性控释, 从而实现智能化 的控制 ${ }^{[107]}$. SR-MIPs的响应聚合单体的活性基团的结 构和性质会随着外界环境的物理化学或生物刺激而发 生可逆改变, 从而使整个SR-MIPs的印迹性能随着外界 刺激改变而产生规律性变化. 因此, SR-MIPs在药物输 送、缓控释等领域大有可为 ${ }^{[108,109]}$.

SR-MIPs研究较多的响应类型有磁、温度(热)、 光及 $\mathrm{pH}$ 刺激型. 磁性SR-MIPs的制备, 是将磁敏感材料 (如最常用的 $\mathrm{Fe}_{3} \mathrm{O}_{4}$ 纳米球)包封到载体中, 以磁性材料 为核，通过表面印迹技术在其表面制备纳米尺度的印 迹聚合物层. 磁SR-MIPs 可以在外界磁场作用下进行定 向移动，不仅能够解决传统方法制备的MIPs结合容量 低、模板分子难以洗脱、传递速率慢等问题，并且具 有容易快速分离和可重复利用的效果. 温敏SR-MIPs (又称热敏SR-MIPs)的制备，是在聚合体系中除了加人 常规的功能单体外, 再加人温度敏感型功能单体(如 $N$ 异丙基丙烯酰胺)为共单体; 可达到通过温度升降来控 释目标分子的作用, 并能够调节聚合物的水溶性. 光敏 SR-MIPs的制备, 是在聚合物的主链中引人光敏基团即 光响应功能单体(如偶氮苯、二苯乙烯等), 在光照条件 下, 光敏基团会发生结构、极性等变化, 并进一步引起 整个聚合物产生形态变化，从而利用光照达到分子控 释的目的，避免使用大量的有机溶剂对模板分子进行 洗脱. $\mathrm{pH}$ 型SR-MIPs的制备主要使用带有羧基或氨基 的可电离的功能单体，通常 $\mathrm{pH}$ 敏感型单体中含有弱酸 性/弱碱性基团(如羧基、磺酸基、氨基等)，随着环境 $\mathrm{pH} /$ 离子强度的改变，这些基团容易发生电离，造成单 体分子内外离子浓度改变，并导致大分子链段间氢键 的解离, 引起不连续的溶胀体积变化或溶解度的改变, 从而达到控释目标分子的目的.

目前, SR-MIPs的研究已从单一刺激响应的智能水 凝胶，逐步发展到生物相容性好、双重甚至多重刺激 响应智能聚合物的研究. 多种刺激响应协同作用使得 SR-MIPs具有更好的可控性、特异性和灵敏性, 但是将 两个或多个刺激响应元素结合到一起时，必须考虑其 合理有效的结合方式和合成方法．以简单共聚方式将
响应元素结合, 会带来相互干扰和信号消减问题. 多个 刺激响应元素有效组合要考虑其协同效果——使每一 种刺激响应信号优势充分发挥，一般还要比单一刺激 效果好得多, 否则多个刺激响应就失去了意义. 磁、热 双响应SR-MIPs的制备一般是以磁性微球为核支撑材 料，通过表面印迹技术制备含有温度敏感型功能单 体、模板、交联剂的印迹壳; 合成时，注意对核修饰的 层次性、先后顺序，并且需要结合活性聚合技术、点 击化学技术等. 例如, 本课题组 ${ }^{[13]}$ 制备了亲水性温、磁 双响应的印迹聚合物(WC-TMMIPs), 选择 $35^{\circ} \mathrm{C}$ 作为实 验温度，将其作为MSPE吸附剂和SPE填料，用于海水 中双酚 $\mathrm{A}$ 的选择性萃取。如图 10所示，首先合成 $\mathrm{Fe}_{3} \mathrm{O}_{4}$, 利用 $\mathrm{SiO}_{2}$ 对 $\mathrm{Fe}_{3} \mathrm{O}_{4}$ 进行包覆，将包覆材料 $\left(\mathrm{Fe}_{3} \mathrm{O}_{4} @ \mathrm{SiO}_{2}\right)$ 作为核支撑材料, 采用表面印迹技术, 使用 $N$-异丙基丙 烯酰胺(NIPAM) 为温敏功能单体、MAA为聚合功能单 体, 结合RAFT技术制备了MIPs层, 洗脱模板后, 再次结 合RAFT技术, 以甲基丙烯酸着㔯乙酯(HEMA)为单体, 在 印迹聚合物的表面引人亲水性刷. 该WC-TMMIPs材料 具有优良的热敏性和简单快速的磁响应性，同时还具 有良好的亲水性(图10). 光、热或 $\mathrm{pH}$ 多响应SR-MIPs制 备时, 除了将多种响应型单体共聚制备印迹材料外 ${ }^{[110]}$, 一般以一种响应单体参与印迹，将制备好的印迹材料 结合活性聚合技术在材料表面引入温度响应/ $\mathrm{pH}$ 聚合 物刷，既赋予材料刺激响应性，又增加了材料的亲水 性 ${ }^{[111,112]}$. 例如，张会旗课题组 ${ }^{[112]}$ 以RAFT技术首先合 成“活性”聚合物，以此为核支撑材料，然后以偶氮苯类 为光响应功能单体，通过表面引发RAFT技术制备了光 响应印迹层，洗脱模板后，再通过活性聚合，以 $N$-异丙 基丙烯酰胺为温度响应单体、甲基丙烯酸二甲胺乙酯 为 $\mathrm{pH}$ 响应单体，在核壳印迹材料表面同时嫁接上温度 和 $\mathrm{pH}$ 响应刷. 该复合材料除具有光、热和 $\mathrm{pH}$ 响应外, 通过嫁接温度和 $\mathrm{pH}$ 响应刷，材料的亲水性也得到很大 改善.

通过发展单、双/多重响应SR-MIPs，既有助于克 服传统MIPs的模板分子洗脱困难、水相识别能力差、 结合容量低等问题，也有助于发展新型的高效、智 能、无污染的MIPs. 具有智能、经济、环保优势的刺 激响应应用，有望推动MIPs制备技术和策略的进一步 发展，加速MIPs在药物输送和缓控释领域的应用，及 为已广泛开展的MIPs样品前处理和传感应用助力. 与 此同时, 对刺激响应印迹来说, 亟须应对几个主要的挑 战: 如, 设计合成新的响应功能单体; 探索新的刺激响 
(a)
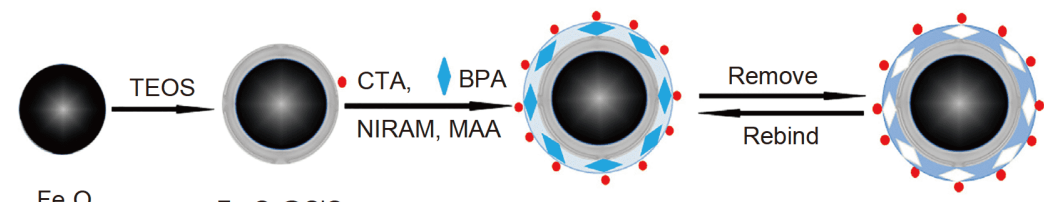

$\mathrm{Fe}_{3} \mathrm{O}_{4}$

$\mathrm{Fe}_{3} \mathrm{O}_{4} @ \mathrm{SiO}_{2}$
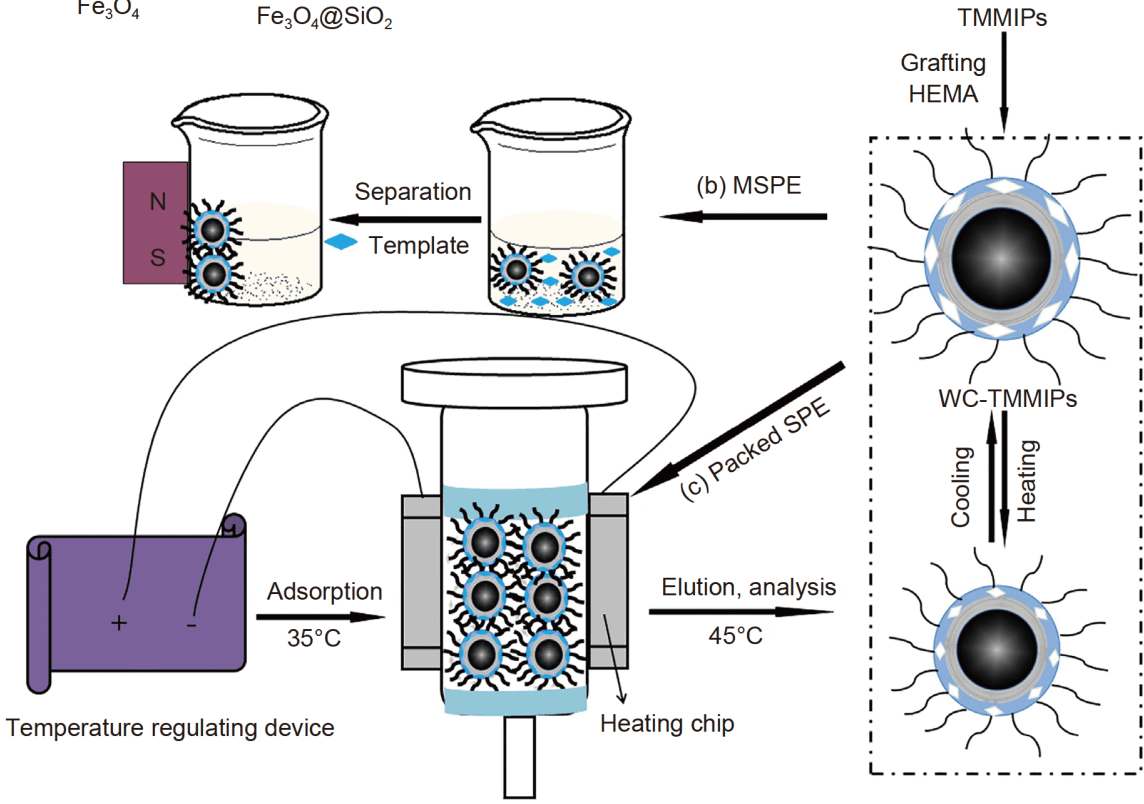

图 10 (网络版彩色)亲水性温、磁双重响应(WC-TMMIPs)的制备过程(a)、温度调控下的收缩/溶胀性能(虚线框图内)及MSPE(b)和SPE(c)应 用 $^{[13]}$

Figure 10 (Color online) Schematic illustration of the preparation process (a), temperature-regulated shrinking/swelling behaviors (in the dotted box) and the applications to MSPE (b) and packed SPE (c) of WC-TMMIPs ${ }^{[13]}$

应系统; 发展多响应SR-MIPs中需要注意巧妙合理的组 合，确保多响应元素提供最佳协同效果; 环境友好型 SR-MIPs如何更好地应用于水体与生理环境中.

\section{4 展望}

为了使印迹聚合材料在实际应用中获得良好的使 用效果, 各种印迹技术和策略需要联合使用. 增加印迹 材料的选择性、传质速率和吸附容量始终是要解决的 核心问题. 为了解决选择性问题, 还要不断探索与模板
分子结合的专一性单体而不是普适性单体，设计和合 成新的专一性单体是提高MIPs选择性的有效方法，并 且在设计合成过程中, 辅以理论计算(计算机模拟等)能 大大节省时间、提高成功率．传质速率的提高除了将 印迹材料制备成微纳结构外，提供介孔结构也是不错 的选择. 吸附容量的提高在于充分利用印迹材料的比 表面积. 另外, 各种新型的聚合物制备技术及其他领域 的先进技术仍然需要不断借鉴和应用到印迹材料的制 备中.

\section{参考文献}

1 Polyakov M V, Khim Z. Adsorption properties of silica gel and its structure. Zhur Fiz Khim, 1931, 2: 799-805

2 Dickey F H. The preparation of specific adsorbents. Proc Natl Acad Sci USA, 1949, 35: 227-229

3 Wulff G, Sarhan A. Use of polymers with enzyme-analogous structures for resolution of racemates. Angew Chem Int Ed, 1972, 11: 341-344

4 Wulff G, Sarhan A, Zabrocki K. Enzyme-analogue built polymers and their use for the resolution of racemates. Tetrahedron Lett, 1973, 14: 43294332

5 Vlatakis G, Andersson L I, Müller R, et al. Drug assay using antibody mimics made by molecular imprinting. Nature, 1993, 361: 645-647

6 Chen L, Xu S, Li J. Recent advances in molecular imprinting technology: Current status, challenges and highlighted applications. Chem Soc Rev, 2011, 40: 2922-2942

7 Chen L, Wang X, Lu W, et al. Molecular imprinting: Perspectives and applications. Chem Soc Rev, 2016, 45: 2137-2211

$8 \mathrm{Li} \mathrm{Y,} \mathrm{Li} \mathrm{X,} \mathrm{Dong} \mathrm{C,} \mathrm{et} \mathrm{al.} \mathrm{Selective} \mathrm{recognition} \mathrm{and} \mathrm{removal} \mathrm{of} \mathrm{chlorophenols} \mathrm{from} \mathrm{aqueous} \mathrm{solution} \mathrm{using} \mathrm{molecularly} \mathrm{imprinted} \mathrm{polymer}$ 
prepared by reversible addition-fragmentation chain transfer polymerization. Biosens Bioelectron, 2009, 25: 306-312

$9 \mathrm{Zu} \mathrm{B}$, Pan G, Guo X, et al. Preparation of molecularly imprinted polymer microspheres via atom transfer radical precipitation polymerization. J Polym Sci Pol Chem, 2009, 47: 3257-3270

10 Beyazit S, Tse Sum Bui B, Haupt K, et al. Molecularly imprinted polymer nanomaterials and nanocomposites by controlled/living radical polymerization. Prog Polym Sci, 2016, 62: 1-21

11 Bompart M, Haupt K. Molecularly imprinted polymers and controlled/living radical polymerization. Aust J Chem, 2009, 62: 751-761

12 Garcia-Soto M J, Haupt K, Gonzato C. Synthesis of molecularly imprinted polymers by photo-iniferter polymerization under visible light. Polym Chem, 2017, 8: 4830-4834

13 Wu X, Wang X, Lu W, et al. Water-compatible temperature and magnetic dual-responsive molecularly imprinted polymers for recognition and extraction of bisphenol A. J Chromatogr A, 2016, 1435: 30-38

14 Wang Y, Jiao S Q, Chen X L, et al. Preparation and detection of progesterone by molecularly imprinted film based on reversible addition fragmentation chain transfer polymerization (in Chinese). Chin Sci Bull, 2018, 63: 68-77 [王洋, 焦少勤, 陈小丽, 等. 基于可逆加成-断裂链转 移聚合的孕酮分子印迹膜制备与检测. 科学通报, 2018, 63: 68-77]

15 Kolb H C, Finn M G, Sharpless K B. Click chemistry: Diverse chemical function from a few good reactions. Angew Chem Int Ed, 2001, 40: 2000-2021

16 Chang L, Li Y, Chu J, et al. Preparation of core-shell molecularly imprinted polymer via the combination of reversible addition-fragmentation chain transfer polymerization and click reaction. Anal Chim Acta, 2010, 680: 65-71

17 Bonomi P, Attieh M D, Gonzato C, et al. A new versatile water-soluble iniferter platform for the preparation of molecularly imprinted nanoparticles by photopolymerisation in aqueous media. Chem Eur J, 2016, 22: 10150-10154

18 Awino J K, Gunasekara R W, Zhao Y. Selective recognition of d-aldohexoses in water by boronic acid-functionalized, molecularly imprinted cross-linked micelles. J Am Chem Soc, 2016, 138: 9759-9762

19 Fan D, Li H, Shi S, et al. Hollow molecular imprinted polymers towards rapid, effective and selective extraction of caffeic acid from fruits. J Chromatogr A, 2016, 1470: 27-32

20 Ostovan A, Ghaedi M, Arabi M, et al. Hollow porous molecularly imprinted polymer for highly selective clean-up followed by influential preconcentration of ultra-trace glibenclamide from bio-fluid. J Chromatogr A, 2017, 1520: 65-74

21 Gong C B, Yang Y Z, Yang Y H, et al. Photoresponsive hollow molecularly imprinted polymer for the determination of trace bisphenol A in water. J Colloid Interface Sci, 2016, 481: 236-244

22 Zhang Z, Zhang X, Niu D, et al. Highly efficient and selective removal of trace lead from aqueous solutions by hollow mesoporous silica loaded with molecularly imprinted polymers. J Hazard Mater, 2017, 328: 160-169

23 Fan D, Jia L, Xiang H, et al. Synthesis and characterization of hollow porous molecular imprinted polymers for the selective extraction and determination of caffeic acid in fruit samples. Food Chem, 2017, 224: 32-36

24 Gong C B, Wei Y B, Liu L T, et al. Photoresponsive hollow molecularly imprinted polymer for trace triamterene in biological samples. Mater Sci Eng C, 2017, 76: 568-578

25 Xiang H, Peng M, Li H, et al. High-capacity hollow porous dummy molecular imprinted polymers using ionic liquid as functional monomer for selective recognition of salicylic acid. J Pharmaceut Biomed, 2017, 133: 75-81

$26 \mathrm{Hu}$ Y, Xia Q, Huang W, et al. Boronate-modified hollow molecularly imprinted polymers for selective enrichment of glycosides. Microchim Acta, 2017, 185: 46

27 Poma A, Guerreiro A, Whitcombe M J, et al. Solid-phase synthesis of molecularly imprinted polymer nanoparticles with a reusable template"plastic antibodies". Adv Funct Mater, 2013, 23: 2821-2827

28 Garcia-Mutio D, Gomez-Caballero A, Guerreiro A, et al. Solid-phase synthesis of imprinted nanoparticles grafted on gold substrates for voltammetric sensing of 4-ethylphenol. Sensor Actuat B Chem, 2016, 236: 839-848

29 Canfarotta F, Czulak J, Betlem K, et al. A novel thermal detection method based on molecularly imprinted nanoparticles as recognition elements. Nanoscale, 2018, 10: 2081-2089

30 Mourão C A, Bokeloh F, Xu J, et al. Dual-oriented solid-phase molecular imprinting: Toward selective artificial receptors for recognition of nucleotides in water. Macromolecules, 2017, 50: 7484-7490

$31 \mathrm{Xu}$ J, Haupt K, Tse Sum Bui B. Core-shell molecularly imprinted polymer nanoparticles as synthetic antibodies in a sandwich fluoroimmunoassay for trypsin determination in human serum. ACS Appl Mater Interfaces, 2017, 9: 24476-24483

32 Chen L, Muhammad T, Yakup B, et al. New immobilisation protocol for the template used in solid-phase synthesis of MIP nanoparticles. Appl Surface Sci, 2017, 406: 115-121

33 Ashley J, Feng X, Halder A, et al. Dispersive solid-phase imprinting of proteins for the production of plastic antibodies. Chem Commun, 2018, 54: $3355-3358$

34 Fu J, Chen L, Li J, et al. Current status and challenges of ion imprinting. J Mater Chem A, 2015, 3: 13598-13627

35 Song X, Li J, Xu S, et al. Determination of 16 polycyclic aromatic hydrocarbons in seawater using molecularly imprinted solid-phase extraction 
coupled with gas chromatography-mass spectrometry. Talanta, 2012, 99: 75-82

$36 \mathrm{Lu} \mathrm{W}$, Wang X, Wu X, et al. Multi-template imprinted polymers for simultaneous selective solid-phase extraction of six phenolic compounds in water samples followed by determination using capillary electrophoresis. J Chromatogr A, 2017, 1483: 30-39

37 Ostovan A, Ghaedi M, Arabi M, et al. Hydrophilic multitemplate molecularly imprinted biopolymers based on a green synthesis strategy for determination of b-family vitamins. ACS Appl Mater Interfaces, 2018, 10: 4140-4150

$38 \mathrm{Xu} \mathrm{Y,} \mathrm{Tang} \mathrm{Y,} \mathrm{Zhao} \mathrm{Y,} \mathrm{et} \mathrm{al.} \mathrm{Bifunctional} \mathrm{monomer} \mathrm{magnetic} \mathrm{imprinted} \mathrm{nanomaterials} \mathrm{for} \mathrm{selective} \mathrm{separation} \mathrm{of} \mathrm{tetracyclines} \mathrm{directly} \mathrm{from} \mathrm{milk}$ samples. J Colloid Interface Sci, 2018, 515: 18-26

39 Liao S L, Chen S Y, Liu Q L, et al. Preparation and application of ornidazole magenetic imprinted polymers with dual functional monomers (in Chinese). Chi J Anal Chem, 2018, 46: 100-106 [廖素兰, 陈少云, 刘奇琳, 等. 双功能单体法制备磁性奥硝唑分子印迹聚合物及应用. 分析化 学, 2018, 46: 100-106]

40 Suo D, Wang R, Wang P, et al. Pseudo template molecularly imprinted polymer for determination of 14 kind of $\beta$-agonists in animal urine by ultra-high-performance liquid chromatography-tandem mass spectrometry. J Chromatogr A, 2017, 1526: 23-30

41 Özer E T, Osman B, Yazıcı T. Dummy molecularly imprinted microbeads as solid-phase extraction material for selective determination of phthalate esters in water. J Chromatogr A, 2017, 1500: 53-60

42 Ndunda E N, Mizaikoff B. Synthesis of stationary phases that provide group recognition for polychlorinated biphenyls by porogenic fragment template imprinting. J Sep Sci, 2016, 39: 939-946

43 Marć M, Panuszko A, Namieśnik J, et al. Preparation and characterization of dummy-template molecularly imprinted polymers as potential sorbents for the recognition of selected polybrominated diphenyl ethers. Anal Chim Acta, 2018, 1030: 77-95

$44 \mathrm{Hu} \mathrm{X}, \mathrm{Wu} \mathrm{X}$, Yang F, et al. Novel surface dummy molecularly imprinted silica as sorbent for solid-phase extraction of bisphenol A from water samples. Talanta, 2016, 148: 29-36

45 Song Y P, Li N, Zhang H C, et al. Dummy template molecularly imprinted polymer for solid phase extraction of phenothiazines in meat based on computational simulation. Food Chem, 2017, 233: 422-428

46 Guo P, Zhang J, Chen X, et al. Preparation of dummy template-imprinted polymers for the rapid extraction of nonsteroidal anti-inflammatory drugs residues in aquatic environmental samples. Biomed Chromatogr, 2018, 32: e4193

47 Lu C, Tang Z, Gao X X, et al. Computer-aided design of magnetic dummy molecularly imprinted polymers for solid-phase extraction of ten phthalates from food prior to their determination by GC-MS/MS. Microchim Acta, 2018, 185: 373

48 Zhang M, He J, Shen Y, et al. Application of pseudo-template molecularly imprinted polymers by atom transfer radical polymerization to the solid-phase extraction of pyrethroids. Talanta, 2018, 178: 1011-1016

49 Zhang Y Z, Zhang J W, Wang C Z, et al. Polydopamine-coated magnetic molecularly imprinted polymers with fragment template for identification of Pulsatilla saponin metabolites in rat Feces with UPLC-Q-TOF-MS. J Agric Food Chem, 2018, 66: 653-660

50 He X, Chen J, Wang J, et al. Multipoint recognition of domoic acid from seawater by dummy template molecularly imprinted solid-phase extraction coupled with high-performance liquid chromatography. J Chromatogr A, 2017, 1500: 61-68

51 Quaglia M, Chenon K, Hall A J, et al. Target analogue imprinted polymers with affinity for folic acid and related compounds. J Am Chem Soc, 2001, 123: 2146-2154

$52 \mathrm{Xu} \mathrm{S}$, Lu H, Li J, et al. Dummy molecularly imprinted polymers-capped CdTe quantum dots for the fluorescent sensing of 2,4,6-trinitrotoluene. ACS Appl Mater Interfaces, 2013, 5: 8146-8154

53 Dan L, Wang H F. Mn-doped ZnS quantum dot imbedded two-fragment imprinting silica for enhanced room temperature phosphorescence probing of domoic acid. Anal Chem, 2013, 85: 4844-4848

54 Huang S, Xu J, Zheng J, et al. Synthesis and application of magnetic molecularly imprinted polymers in sample preparation. Anal Bioanal Chem, 2018, 410: 3991-4014

55 Ansari S. Application of magnetic molecularly imprinted polymer as a versatile and highly selective tool in food and environmental analysis: Recent developments and trends. TrAC Trends Anal Chem, 2017, 90: 89-106

56 Pan X, He X, Liu Z. Molecularly imprinted mesoporous silica nanoparticles for specific extraction and efficient identification of Amadori compounds. Anal Chim Acta, 2018, 1019: 65-73

57 Liu Z, He H. Synthesis and applications of boronate affinity materials: From class selectivity to biomimetic specificity. Accounts Chem Res, 2017, 50: $2185-2193$

58 Chen Y, Li D, Bie Z, et al. Coupling of phosphate-imprinted mesoporous silica nanoparticles-based selective enrichment with matrix-assisted laser desorption ionization-time-of-flight mass spectrometry for highly efficient analysis of protein phosphorylation. Anal Chem, 2016, 88: 14471454

59 Bi X, Liu Z. Enzyme activity assay of glycoprotein enzymes based on a boronate affinity molecularly imprinted 96-well microplate. Anal Chem, 2014, 86: 12382-12389

60 Li L, Lu Y, Bie Z, et al. Photolithographic boronate affinity molecular imprinting: A general and facile approach for glycoprotein imprinting. Angew Chem Int Ed, 2013, 52: 7451-7454 
61 Wang S, Ye J, Bie Z, et al. Affinity-tunable specific recognition of glycoproteins via boronate affinity-based controllable oriented surface imprinting. Chem Sci, 2014, 5: 1135-1140

62 Bie Z, Chen Y, Ye J, et al. Boronate-affinity glycan-oriented surface imprinting: A new strategy to mimic lectins for the recognition of an intact glycoprotein and its characteristic fragments. Angew Chem Int Ed, 2015, 54: 10211-10215

63 Bie Z, Xing R, He X, et al. Precision imprinting of glycopeptides for facile preparation of glycan-specific artificial antibodies. Anal Chem, 2018, 90: 9845-9852

64 Xing R, Wang S, Bie Z, et al. Preparation of molecularly imprinted polymers specific to glycoproteins, glycans and monosaccharides via boronate affinity controllable-oriented surface imprinting. Nat Protoc, 2017, 12: 964-987

65 Bi X, Liu Z. Facile preparation of glycoprotein-imprinted 96-well microplates for enzyme-linked immunosorbent assay by boronate affinity-based oriented surface imprinting. Anal Chem, 2014, 86: 959-966

66 Chen Y, Li X, Yin D, et al. Dual-template docking oriented molecular imprinting: A facile strategy for highly efficient imprinting within mesoporous materials. Chem Commun, 2015, 51: 10929-10932

67 Zaidi S A. Utilization of an environmentally-friendly monomer for an efficient and sustainable adrenaline imprinted electrochemical sensor using graphene. Electrochim Acta, 2018, 274: 370-377

68 Huang J, Wu Y, Cong J, et al. Selective and sensitive glycoprotein detection via a biomimetic electrochemical sensor based on surface molecular imprinting and boronate-modified reduced graphene oxide. Sensor Actuat B Chem, 2018, 259: 1-9

69 Rao H, Zhao X, Liu X, et al. A novel molecularly imprinted electrochemical sensor based on graphene quantum dots coated on hollow nickel nanospheres with high sensitivity and selectivity for the rapid determination of bisphenol S. Biosens Bioelectron, 2018, 100: 341-347

70 Zhao X, Hu W, Wang Y, et al. Decoration of graphene with 2-aminoethanethiol functionalized gold nanoparticles for molecular imprinted sensing of erythrosine. Carbon, 2018, 127: 618-626

71 Luo J, Huang J, Cong J, et al. Double recognition and selective extraction of glycoprotein based on the molecular imprinted graphene oxide and boronate affinity. ACS Appl Mater Interfaces, 2017, 9: 7735-7744

72 Gautam V, Singh K P, Yadav V L. Polyaniline/MWCNTs/starch modified carbon paste electrode for non-enzymatic detection of cholesterol: Application to real sample (cow milk). Anal Bioanal Chem, 2018, 410: 2173-2181

73 Munawar A, Tahir M A, Shaheen A, et al. Investigating nanohybrid material based on 3D CNTs@Cu nanoparticle composite and imprinted polymer for highly selective detection of chloramphenicol. J Hazard Mater, 2018, 342: 96-106

74 Yang L, Xu B, Ye H, et al. A novel quercetin electrochemical sensor based on molecularly imprinted poly(para-aminobenzoic acid) on 3D Pd nanoparticles-porous graphene-carbon nanotubes composite. Sensor Actuat B Chem, 2017, 251: 601-608

75 Wang Z, Qian Y, Wei X, et al. An “on-off” electrochemiluminescence biosensor based on molecularly imprinted polymer and recycling amplifications for determination of dopamine. Electrochim Acta, 2017, 250: 309-319

76 Yang Y, Fang G, Wang X, et al. Imprinting of molecular recognition sites combined with $\pi$-donor-acceptor interactions using bis-anilinecrosslinked $\mathrm{Au}-\mathrm{CdSe} / \mathrm{ZnS}$ nanoparticles array on electrodes: Development of electrochemiluminescence sensor for the ultrasensitive and selective detection of 2-methyl-4-chlorophenoxyacetic acid. Biosens Bioelectron, 2016, 77: 1134-1143

77 Liu Y, Hu X, Bai L, et al. A molecularly imprinted polymer placed on the surface of graphene oxide and doped with Mn(II)-doped ZnS quantum dots for selective fluorometric determination of acrylamide. Microchim Acta, 2017, 185: 48

78 Fan L, Hao Q, Kan X. Three-dimensional graphite paper based imprinted electrochemical sensor for tertiary butylhydroquinone selective recognition and sensitive detection. Sensor Actuat B Chem, 2018, 256: 520-527

79 Zhang J, Wang C, Niu Y, et al. Electrochemical sensor based on molecularly imprinted composite membrane of poly(o-aminothiophenol) with gold nanoparticles for sensitive determination of herbicide simazine in environmental samples. Sensor Actuat B Chem, 2017, 249: 747-755

80 Yang H, Li L, Ding Y, et al. Molecularly imprinted electrochemical sensor based on bioinspired Au microflowers for ultra-trace cholesterol assay. Biosens Bioelectron, 2017, 92: 748-754

81 Wang H, Yao S, Liu Y, et al. Molecularly imprinted electrochemical sensor based on Au nanoparticles in carboxylated multi-walled carbon nanotubes for sensitive determination of olaquindox in food and feedstuffs. Biosens Bioelectron, 2017, 87: 417-421

82 Wang X, Yu J, Li J, et al. Quantum dots based imprinting fluorescent nanosensor for the selective and sensitive detection of phycocyanin: A general imprinting strategy toward proteins. Sensor Actuat B Chem, 2018, 255: 268-274

83 Li X, Jiao H F, Shi X Z, et al. Development and application of a novel fluorescent nanosensor based on FeSe quantum dots embedded silica molecularly imprinted polymer for the rapid optosensing of cyfluthrin. Biosens Bioelectron, 2018, 99: 268-273

84 Ensafi A A, Nasr-Esfahani P, Rezaei B. Synthesis of molecularly imprinted polymer on carbon quantum dots as an optical sensor for selective fluorescent determination of promethazine hydrochloride. Sensor Actuat B Chem, 2018, 257: 889-896

85 Xu S, Lu H. Mesoporous structured MIPs@CDs fluorescence sensor for highly sensitive detection of TNT. Biosens Bioelectron, 2016, 85: 950956

86 Li X G, Zhang F, Gao Y, et al. Facile synthesis of red emitting 3-aminophenylboronic acid functionalized copper nanoclusters for rapid, selective and highly sensitive detection of glycoproteins. Biosens Bioelectron, 2016, 86: 270-276 
87 Liu J M, Cao F Z, Fang G Z, et al. Upconversion nanophosphor-involved molecularly imprinted fluorescent polymers for sensitive and specific recognition of sterigmatocystin. Polymers, 2017, 9: 299

88 Guo T, Deng Q, Fang G, et al. A double responsive smart upconversion fluorescence sensing material for glycoprotein. Biosens Bioelectron, 2016, 85: 596-602

89 Yang Q, Li J, Wang X, et al. Strategies of molecular imprinting-based fluorescence sensors for chemical and biological analysis. Biosens Bioelectron, 2018, 112: 54-71

90 Liang C, Wang H, He K, et al. A virus-MIPs fluorescent sensor based on FRET for highly sensitive detection of JEV. Talanta, 2016, 160: 360-366

91 Ming W, Wang X, Lu W, et al. Magnetic molecularly imprinted polymers for the fluorescent detection of trace 17 $\beta$-estradiol in environmental water. Sensor Actuat B Chem, 2017, 238: 1309-1315

92 Yu J, Wang X, Kang Q, et al. One-pot synthesis of a quantum dot-based molecular imprinting nanosensor for highly selective and sensitive fluorescence detection of 4-nitrophenol in environmental waters. Environ Sci Nano, 2017, 4: 493-502

93 Wang S, Wen Y, Wang Y, et al. Pattern recognition of cells via multiplexed imaging with monosaccharide-imprinted quantum dots. Anal Chem, 2017, 89: 5646-5652

94 Jia M F, Zhang Z, Yang X B, et al. Design and application of novel molecular imprinting fluorescent sensors (in Chinese). Sci Sin Chim, 2017, 47: 300-314 [贾梦凡, 张忠, 杨兴斌, 等. 新型分子印迹荧光传感器的构建与应用. 中国科学: 化学, 2017, 47: 300-314]

95 Wang X, Yu J, Kang Q, et al. Molecular imprinting ratiometric fluorescence sensor for highly selective and sensitive detection of phycocyanin. Biosens Bioelectron, 2016, 77: 624-630

96 Amjadi M, Jalili R. Molecularly imprinted mesoporous silica embedded with carbon dots and semiconductor quantum dots as a ratiometric fluorescent sensor for diniconazole. Biosens Bioelectron, 2017, 96: 121-126

97 Wei J R, Chen H Y, Zhang W, et al. Ratiometric fluorescence for sensitive and selective detection of mitoxantrone using a MIP@rQDs@SiO 2 fluorescence probe. Senss Actuators B Chem, 2017, 244: 31-37

$98 \mathrm{Li} \mathrm{W}$, Zhang H, Chen S, et al. Synthesis of molecularly imprinted carbon dot grafted $\mathrm{YVO}_{4}$ : $\mathrm{Eu}^{3+}$ for the ratiometric fluorescent determination of paranitrophenol. Sensor Actuat B Chem, 2016, 86: 706-713

99 Wang X, Yu J, Wu X, et al. A molecular imprinting-based turn-on ratiometric fluorescence sensor for highly selective and sensitive detection of 2,4-dichlorophenoxyacetic acid (2,4-D). Biosens Bioelectron, 2016, 81: 438-444

100 Li M, Liu H, Ren X. Ratiometric fluorescence and mesoporous structured imprinting nanoparticles for rapid and sensitive detection 2,4,6trinitrophenol. Biosens Bioelectron, 2017, 89: 899-905

$101 \mathrm{Xu} \mathrm{S}, \mathrm{Lu} \mathrm{H}$. Ratiometric fluorescence and mesoporous structure dual signal amplification for sensitive and selective detection of TNT based on MIP@QD fluorescence sensors. Chem Commun, 2015, 51: 3200-3203

102 Wang X, Yu S, Liu W, et al. Molecular imprinting based hybrid ratiometric fluorescence sensor for the visual determination of bovine hemoglobin. ACS Sens, 2018, 3: 378-385

103 Ye J, Chen Y, Liu Z. A boronate affinity sandwich assay: An appealing alternative to immunoassays for the determination of glycoproteins. Angew Chem Int Ed, 2014, 53: 10386-10389

104 Tu X, Muhammad P, Liu J, et al. Molecularly imprinted polymer-based plasmonic immunosandwich assay for fast and ultrasensitive determination of trace glycoproteins in complex samples. Anal Chem, 2016, 88: 12363-12370

105 Liu J, Yin D, Wang S, et al. Probing low-copy-number proteins in a single living cell. Angew Chem Int Ed, 2016, 55: 13215-13218

106 Muhammad P, Tu X, Liu J, et al. Molecularly imprinted plasmonic substrates for specific and ultrasensitive immunoassay of trace glycoproteins in biological samples. ACS Appl Mater Interfaces, 2017, 9: 12082-12091

107 Xu S, Lu H, Zheng X, et al. Stimuli-responsive molecularly imprinted polymers: Versatile functional materials. J Mater Chem C, 2013, 1: 44064422

$108 \mathrm{Li} \mathrm{W}$, Dong K, Ren J, et al. A $\beta$-lactamase-imprinted responsive hydrogel for the treatment of antibiotic-resistant bacteria. Angew Chem, 2016, 128: $8181-8185$

109 Singh B, Khurana R K, Garg B, et al. Stimuli-responsive systems with diverse drug delivery and biomedical applications: Recent updates and mechanistic pathways. Crit Rev Ther Drug, 2017, 34: 209-255

110 Gao F X, Ma X T, He X W, et al. Smart surface imprinting polymer nanospheres for selective recognition and separation of glycoprotein. Colloid Surface A, 2013, 433: 191-199

111 Li C, Ma Y, Niu H, et al. Hydrophilic hollow molecularly imprinted polymer microparticles with photo- and thermoresponsive template binding and release properties in aqueous media. ACS Appl Mater Interfaces, 2015, 7: 27340-27350

112 Ma Y, Zhang Y, Zhao M, et al. Efficient synthesis of narrowly dispersed molecularly imprinted polymer microspheres with multiple stimuliresponsive template binding properties in aqueous media. Chem Commun, 2012, 48: 6217-6219 


\title{
Advanced preparation technologies and strategies for molecularly imprinted materials
}

\author{
Xiaoyan Wang ${ }^{1,2^{*}}$, Jinhua $\mathrm{Li}^{2}$ \& Lingxin Chen ${ }^{2,3^{*}}$ \\ ${ }^{1}$ School of Pharmacy, Binzhou Medical University, Yantai 264003, China; \\ ${ }^{2}$ CAS Key Laboratory of Coastal Environmental Processes and Ecological Remediation, Yantai Institute of Coastal Zone Research, Chinese Academy of \\ Sciences, Yantai 264003, China; \\ ${ }^{3}$ Laboratory for Marine Biology and Biotechnology, Pilot National Laboratory for Marine Science and Technology, Qingdao 266237, China \\ * Corresponding authors, E-mail: wangxy@yic.ac.cn; 1xchen@yic.ac.cn
}

Molecularly imprinted polymers (MIPs) prepared by molecular imprinting technology (MIT) are polymers with specific recognition sites matching the shape, size and functional groups of template molecules, which can selectively identify and enrich target analytes (template molecules), and have been widely used in sample pretreatment, chemical/biological sensing and other fields. However, in the processes of preparation and applications of MIPs, there are still some problems, such as difficult elution of template molecules, fewer effective recognition sites, low binding capacity, low mass transfer rate and poor recognition in aqueous media. As a multidisciplinary technology, MIT has developed rapidly by borrowing and integrating related advanced technologies/strategies of other fields. Consequently, a variety of new imprinting technologies and strategies have continuously emerged, which not only effectively solve the abovementioned problems but also push forwards the development of novel MIPs and widen their applications. In this paper, orienting the applications of MIPs in sample pretreatment, sensors and stimuli responses, some advanced preparation technologies and strategies for MIPs materials are highlighted, including ingenious imprinting technologies (surface imprinting, nanoimprinting; controlled/living polymerization, solid-phase synthesis, etc.), special imprinting strategies (multi-template/monomer imprinting, dummy imprinting, boronate affinity imprinting, etc.), and stimuli-responsive imprinting (magnetic, temperature, $\mathrm{pH}$ responsive, etc.). Fundamental features of the advanced imprinting technologies/strategies and their utilizations for MIPs preparations along with representative applications are described in details, involving important issues and research challenges.

Firstly, a comprehensive overview of main imprinting technologies and strategies for MIPs preparation in sample pretreatment application is provided. In this regard, MIPs are used as selective adsorbents of various extraction technologies such as solid-phase extraction (SPE), dispersive SPE and magnetic SPE. Aiming at high selectivity and high adsorption capacity, the MIPs should have ideal morphology, uniform size and excellent surface properties. Besides conventional preparative methods, it is required to introduce new imprinting technologies and strategies, mainly including the ingenious imprinting technologies of surface imprinting, nanoimprinting, controlled/living free radical polymerization (CLRP), click chemistry, hollow porous polymer synthesis technology and solid-phase synthesis, and the special imprinting strategies of multitemplate/monomer imprinting, dummy/segment imprinting, magnetic material and boronate affinity imprinting. Surface imprinting and nanoimprinting technologies are usually adopted by coupling with the abovementioned imprinting technologies and strategies.

Secondly, advanced imprinting technologies and strategies for the construction of MIPs-based sensors are summarized. For the sensors, the MIPs as recognition elements can specifically bind target analytes and as transduction elements can generate output signals for detection. Typically, the output detection signals can be classified into three types, electrochemical, optical and piezoelectric types according to the transduction mechanism; molecular imprinting based electrochemistry, fluorescence and surface enhanced Raman scattering sensors are the research hotspots. For sensing applications, it is necessary to consider the main parameters such as response time, linear dynamic range, sensitivity, selectivity and reproducibility. Therefore, the MIPs should have excellent interface properties by employing appropriate preparative technologies and strategies. Nanoimprinting, surface imprinting and composite material imprinting strategy have become the preferences. Herein, constructions of molecular imprinting fluorescence sensors are emphatically introduced, especially ratiometric fluorescence ones.

Thirdly, the imprinting technologies and strategies orienting stimuli-responsive application are briefly introduced, for preparing stimuliresponsive MIPs (SR-MIPs) with specific recognition ability toward targeted molecules under stimuli-regulation and thereby achieving intelligent control. SR-MIPs are able to sensitively respond to specific external physicochemical/biological stimuli with a considerable and reversible change in their properties, such as molecular chain structure, solubility, swelling or dissociation behavior, resulting in regular changes of imprinting properties. The most reported magnetic, temperature, photonic and $\mathrm{pH}$ sensitive SR-MIPs and their dual or multi stimuli responsive SR-MIPs are reviewed. The rapid development of smart ecofriendly SR-MIPs and their stimuli-responsive application will accelerate the drug delivery application and assist the widely carried out sample pretreatment and sensors applications.

Lastly, future perspectives of MIT and MIPs are proposed. In order to solve the core issues of selectivity, mass transfer rate and adsorption capacity of MIPs, it is imperative to rationally combine various imprinting technologies and strategies. The ingenious fusion of MIT and various advanced technologies should be continuously strengthened to promote the preparation of MIPs materials.

molecularly imprinted polymers, imprinting technology, imprinting strategy, sample pretreatment, sensor, stimuliresponsive imprinting

doi: 10.1360/N972018-00964 